\title{
Linx
}

Revue des linguistes de l'université Paris X Nanterre

$77 \mid 2018$

Regards croisés sur le futur en français et dans différentes langues romanes

\section{Quelques valeurs énonciatives du futur I en ancien et moyen français}

Evelyne Oppermann-Marsaux et Gabriella Parussa

\section{CpenEdition}

Journals

Édition électronique

URL : http://journals.openedition.org/linx/2758

DOI : $10.4000 /$ linx.2758

ISSN : 2118-9692

Éditeur

Presses universitaires de Paris Nanterre

Édition imprimée

Date de publication : 30 octobre 2018

Pagination : 117-142

ISBN : 978-2-84016-329-9

ISSN : 0246-8743

Référence électronique

Evelyne Oppermann-Marsaux et Gabriella Parussa, «Quelques valeurs énonciatives du futur I en ancien et moyen français », Linx [En ligne], 77 | 2018, mis en ligne le 30 avril 2019, consulté le 03 mai 2019. URL : http://journals.openedition.org/linx/2758 ; DOI : 10.4000/linx.2758

Ce document a été généré automatiquement le 3 mai 2019.

Département de Sciences du langage, Université Paris Ouest 


\title{
Quelques valeurs énonciatives du futur I en ancien et moyen français
}

\author{
Evelyne Oppermann-Marsaux et Gabriella Parussa
}

\section{Introduction}

1 S'il se place dans une perspective historique, le linguiste peut avoir l'impression que tout a été dit au sujet de l'origine et de l'évolution morphologique de ce tiroir verbal. On sait et l'accord est presque unanime là-dessus ${ }^{1}$ - que le futur des langues romanes est issu d'une périphrase verbale, rare en latin classique mais bien attestée en latin tardif, formée avec le verbe avoir, conjugué au présent de l'indicatif, et l'infinitif du verbe. Si à l'origine le verbe avoir garde son sémantisme propre (i.e. possession), il devient vite un simple auxiliaire et cette périphrase, d'abord modale, se grammaticalise progressivement et devient un moyen pour exprimer la futurité d'un évènement par rapport au moment de l'énonciation'.

2 Il est couramment admis aussi que le futur conserve encore en français moderne (FM) des valeurs modales : les grammaires fournissent même des listes, plus ou moins exhaustives, de toutes ces valeurs; atténuation, conjecture et indignation sont les plus courantes, à côté des futurs dits injonctif, gnomique, historique et rétrospectif. Les étiquettes sont nombreuses et elles varient en fonction du type d'analyse et du cadre théorique choisi.

3 Cependant, un examen attentif de la bibliographie sur les valeurs du futur révèle la fragilité de ce consensus aussi bien sur les valeurs primitives de la périphrase latine (modale, temporelle, les deux à la fois ?) que sur la modalité et le rythme selon lesquels l'évolution sémantique a eu lieu, d'abord dans l'histoire du latin, ensuite des langues romanes et du français en particulier. De la même manière, on s'interroge encore sur la priorité entre valeurs temporelles et valeurs modales du futur et sur la persistance ou pas des valeurs temporelles dans les emplois modaux. L'identification et la dénomination des valeurs épistémiques soulèvent de nombreuses questions et fait toujours l'objet de débats, tout comme la concurrence en FM entre futur simple et futur périphrastique. 
4 Au vu de ces questions sur les valeurs du futur qui n'ont pas encore reçu de réponse, il nous a paru intéressant d'analyser la situation pendant la période médiévale pour étudier quelques aspects de l'évolution de ce tiroir verbal et notamment de son sémantisme. Le cadre réduit de cette contribution nous permettra seulement de présenter quelques exemples intéressants relevés dans un corpus de textes narratifs et dramatiques en ancien français (AF) et en moyen français (MF) et, en premier lieu, les occurrences qui sont liées à des situations d'énonciation particulières. Tous les emplois retenus méritent notre attention, soit qu'ils ne correspondent pas aux descriptions proposées par les grammaires de l'ancienne langue, soit qu'ils posent des problèmes de traduction en FM, étant donné que le maintien du futur ne serait pas envisageable dans la langue actuelle.

5 En effet, si la majorité des grammaires historiques consacrent de longs développements à l'identification et à l'explication des changements phonético-morphologiques qui ont touché la forme périphrastique lors du passage du latin aux langues romanes, presque aucune ne propose une étude approfondie et précise de l'évolution sémantique du futur du latin à l'AF, au MF et au FM. L'étude de M. Wilmet (1970) est la seule exception, car elle consacre plusieurs pages à l'analyse d'occurrences extraites de textes dramatiques des $15^{\mathrm{e}}$ et $16^{\mathrm{e}}$ siècles. Il faut donc se tourner vers les études les plus récentes en sémantique verbale pour trouver à la fois des tentatives d'explication et une énumération des valeurs possibles du futur, bien que ces études adoptent rarement une perspective diachronique ${ }^{3}$.

Il a rapidement été évident pour nous que les interprétations des formes de futur proposées par les manuels et les grammaires, malgré leur caractère détaillé, ne permettaient pas d'expliquer plusieurs occurrences relevées dans les textes de notre corpus. En particulier, les formes de futur qui ont partie liée avec l'énonciation, nombreuses dans notre corpus de textes dramatiques et narratifs à forte composante dialogale, semblent résister aux analyses les plus courantes qui distinguent principalement les valeurs proprement temporelles des valeurs autres (injonctive et d'atténuation surtout).

7 Il nous a donc paru intéressant de rouvrir ce dossier et de faire part des résultats de notre enquête. Ceux-ci devraient en effet permettre de remettre en question certaines affirmations trop rapides et/ou superficielles sur les valeurs associées au futur en français médiéval qui ne rendent pas compte du fonctionnement réel de ce tiroir verbal.

8 Nous avons travaillé sur un corpus de textes en AF et en MF, composés entre le $12^{\mathrm{e}}$ siècle et le $16^{\mathrm{e}}$ siècle. Dans le but de mettre en évidence les valeurs énonciatives du futur, notre choix s'est porté sur des textes exclusivement composés de dialogues (ou de monologues), à savoir le théâtre, et sur des textes dans lesquels le dialogue occupe une place importante, comme les récits en vers en $\mathrm{AF}$ et les nouvelles et autres types de discours narratif du $15^{e}$ siècle. Notre corpus comprend donc des récits et des œuvres dramatiques en vers et en prose. Ces textes ont été dépouillés à partir de la base Frantext (pour ce qui est de l'AF) ${ }^{4}$ et du Corpus de Dialogues en français en cours de constitution au sein de l'équipe Clesthia (EA 7345) de Paris 3.

9 Voici une liste des textes sélectionnés dans l'ordre chronologique de composition; l'auteur, le titre et la date de composition sont suivis d'un sigle qui permettra d'identifier facilement la source de chaque exemple cité.

Roman d'Eneas, t. 1, 1155 (Eneas)

Marie de France, Lais, 1160-80 (Sont cités dans cet article: Aüstic, Equitan,

Guigemar et Yonec)

Béroul, Roman de Tristan, 1170-90 (Tristan) 
Chrétien de Troyes, Erec et Enide, 1170 (Erec)

Chrétien de Troyes, Cligès, 1176 (Cligès)

Chrétien de Troyes, Lancelot ou le chevalier de la charrette, 1177 (Lancelot)

Chrétien de Troyes, Yvain ou le chevalier au lion, 1177 (Yvain)

Le procès de Jeanne d'Arc, 1431 (d'après l'éd. P. Doncœur), (PJA)

Le Mystère des Actes des Apôtres, $1455 \mathrm{ca}$. (AA)

Les Cent Nouvelles Nouvelles, 1456-67 (CNN)

La Célestine, trad. fr. par Lavardin, 1586 (Célestine)

\section{Les emplois dialogiques du futur} présent : il « se calcule déictiquement ", étant donné qu'il « s'énonce à partir du locuteurénonciateur $\mathrm{E}_{1}$ situé en $\mathrm{t}_{\mathrm{o}}$ " (Azzopardi \& Bres, 2011 : 72).

Dès le français médiéval, nous rencontrons toutefois des occurrences du futur dans lesquelles cette interprétation pose problème. Il s'agit d'emplois dans lesquels le futur semble bien traduire en premier lieu une valeur temporelle d'ultériorité, mais où le calcul de celle-ci ne peut se faire par rapport au moment d'énonciation $t_{o}$ de son locuteurénonciateur.

(1) Turnus se dementot forment,

n'i vendra pas hastivement,

ainçois verra tier jor passé.

Il ariva soz la cité

dont Daumus ses pere ert sire. (Eneas, v. 5837-41)

[Turnus se lamentait beaucoup, il ne reviendra pas rapidement mais verra auparavant passer trois jours. Il acosta près de la cité dont Daumus, son père, était le seigneur.]

Dans (1), par exemple, les futurs vendra et verra figurent à l'intérieur d'une narration au passé, comportant les imparfaits dementot et ert et le passé simple ariva. Comme ces trois formes verbales, ils renvoient par conséquent à des événements situés dans le passé par rapport au moment d'énonciation du narrateur (qui correspond à $E_{1}$ ).

Nous avons alors affaire à des emplois dialogiques du futur. Comme cela a déjà été montré, notamment par Bres (2009) et Azzopardi \& Bres (2011), le futur a un emploi dialogique à chaque fois que le contexte linguistique de l'occurrence implique un dédoublement énonciatif: alors que "le futur, en tant qu'ultérieur du présent, situe habituellement le procès dans l'avenir à partir du seul locuteur-énonciateur $E_{1}$ " (Bres, 2009 : 28), son emploi nous invite, dans ce cas précis, à poser l'existence d'un deuxième énonciateur, que nous appellerons, à la suite de Bres, $\mathrm{e}_{1}$.

14 A l'intérieur des textes littéraires qui nous intéressent dans le cadre de cette étude, ces emplois dialogiques peuvent figurer aussi bien dans le discours du narrateur - où ils correspondent à un futur historique - que dans le discours des personnages (dans le cadre du discours rapporté).

\subsection{Le futur historique ${ }^{5}$}

Le futur historique, également appelé « futur d'anticipation (ou de prospective) » (Riegel, Pellat, Rioul, 1996: 313), apparaît exclusivement dans la narration. Tout comme le présent historique, il s'emploie dans un contexte au passé. Il sert alors «à évoquer des 
faits postérieurs au moment évoqué, en ouvrant une perspective sur les conséquences futures des événements passés » (ibid.). L'ultériorité y est donc calculée par rapport à ce «moment évoqué » du passé, et non par rapport au moment d'énonciation $t_{o} d u$ narrateur ; elle implique toujours une projection du narrateur au temps des événements racontés. L'exemple (1) relève de ce premier emploi dialogique du futur; il en est de même pour (2) :

(2) Tristran se jut an la fullie.

(...)

Endormiz est, ne savoit mie

Que cil eüst perdu la vie

Par qui il dut mort recevoir :

Liez ert, quant en savra le voir.

Governal a la loge vient,

La teste au mort a sa main tient; ;... (Tristan, v. 1729-36)

[Tristan était étendu dans l'abri de feuillages... Il s'était endormi, il ne savait pas que celui qui avait failli le tuer avait perdu la vie: il sera heureux quand il apprendra la vérité. Governal arrive à la loge, il tient la tête du mort dans sa main... ]

A côté de l'imparfait savoit et du passé simple jut, nous relevons ici les futurs $\operatorname{ert}^{6}$ et savra.

Dans (1) comme dans (2), ces futurs nous invitent à poser un énonciateur $e_{1}$, correspondant à la projection du narrateur au temps des événements passés, afin de calculer l'ultérieur (qu'implique l'emploi du futur) par rapport au moment d'énonciation $t_{1}$ de cet énonciateur $e_{1}$. Dans (1), ce moment d'énonciation est contemporain aux lamentations de Turnus; dans (2), il est situé pendant le sommeil de Tristan, avant le retour de Governal.

Cet emploi historique du futur est bien représenté dans les textes narratifs $d u 12^{\mathrm{e}}$ siècle, probablement à cause de la présence, tout aussi importante, du présent historique ${ }^{7}$ dans ces textes, qui rend compte du même type de changement dans le calcul des déictiques. Ces deux temps sont en effet souvent réunis dans le même contexte.

Ainsi, les futurs historiques de (2) ne sont pas suivis d'un retour à une narration au passé - contrairement à ce qui se produit dans (1) ; le récit se poursuit cette fois au présent historique : Governal vient, tient...

Dans d'autres passages, le présent historique est déjà employé dans le contexte gauche du futur historique ; c'est le cas dans (3), avec les présents se colche et s'endort :

(3) El lit se colche, si s'endort.

Hui a trespassé le plus fort ;

ainz la vespree arivera

la u sa guarisun avra. (Guigemar, v. 203-204)

[Il se couche dans le lit et s'endort. A présent le plus dur est passé ; avant le soir il

acostera là ou il trouvera la guérison.]

21 On note dans cet exemple également la présence de l'adverbe de temps hui (aujourd'hui), qui doit être analysé, tout comme les présents qui le précèdent et les futurs de son contexte droit, en relation avec un moment d'énonciation $\mathrm{t}_{1}$ attribué à $\mathrm{e}_{1}$ et situé dans le passé par rapport au moment d'énonciation $t_{0}$ de l'énonciateur $E_{1}$, qui correspond alors au narrateur.

La présence du futur historique dans les romans en vers et les lais relevant de la période de l'AF semble aussi liée à une autre propriété énonciative de ces textes : il s'agit, comme l'a montré Sophie Marnette, de textes « du je $»^{8}$, c'est-à-dire de textes dans lesquels le 
narrateur laisse de nombreuses traces de sa présence, en particulier à travers des déictiques de première personne et des modalités d'énoncé (traduisant sa pensée, sa subjectivité). Dans notre corpus, le futur historique apparait souvent dans le contexte immédiat de telles traces :

(4) De cel cunseil sunt departi.

A las! cum ierent malbailli

cil que l'um vuelt si agaitier

pur els traïr e engignier ! (Yonec, v. 257-60)

[Cette décision prise, ils se sont séparés. Hélas ! quel malheur va les frapper, ceux que l'on veut ainsi espionner pour les trahir et prendre au piège.]

(5) Erec fu foibles et quassez,

et cil fu auques respassez

de ses plaies et de ses cos.

Or fera Erec trop que fos

se tost conuistre ne se fet. (Erec, v. 4965-69)

[Erec était très faible et endolori, alors que l'autre chevalier <Guivert> s'était en partie remis de ses blessures et des coups reçus. A présent Erec va agir en fou s'il ne se fait pas reconnaître immédiatement.]

(6) Erec de ce rien ne savoit

qu'il deüssent sa mort pleidier,

mes Dex li porra bien aidier,

et je cuit que si fera il. (Erec, v. 3418-21)

[Erec ne savait pas qu'ils étaient sur le point de comploter sa mort; mais Dieu pourra bien lui venir en aide, et je suis persuadé qu'il le fera.]

Ainsi, nous relevons, à côté des futurs historiques ierent (4), fera $(5,6)$ et porra (6), le déictique je (6), l'interjection A las! (4) et la forme verbale cuit (6), traduisant respectivement le regret et la conviction du narrateur, ou encore la tournure «faire trop que fos" (5), par laquelle le narrateur donne son opinion sur l'attitude de son personnage.

Les emplois du futur historique semblent plus rares dans les textes en MF, probablement à cause des changements majeurs qui ont touché le genre narratif, surtout pour ce qui est de sa forme, de sa destination et de la présence du narrateur. Les récits en AF sont destinés à une performance orale devant un public d'auditeurs ; le narrateur y occupe une place importante et laisse des traces évidentes de sa présence. Par contre, dans les textes narratifs en MF, le narrateur se fait plus discret, bien qu'il ne disparaisse pas de la scène énonciative, et la versification laisse la place à la prose, destinée de plus en plus souvent à un lecteur individuel. Il est probable aussi que le choix de notre corpus en MF (majoritairement composé de dialogues dramatiques) détermine ce résultat ${ }^{9}$. Une recherche rapide dans la base Frantext a montré que le futur historique existe, mais qu'il faut le chercher soit dans des textes narratifs versifiés dans la lignée des récits des $12^{\mathrm{e}}-13^{\mathrm{e}}$ siècles, soit dans des chroniques où la présence d'une voix qui raconte les faits favorise la présence de ce type de décrochage. En voici deux exemples éclairants :

(7) Apres tout ce, congié pris a

Dou duc et de la compaingnie.

Si se parti à chiere lie;

Ne mais gueres n'arrestera,

Jusques en Venise sera.

Or deviseray son chemin

Par escript en ce parchemin.

Il s' en ala en Quarateinne,

Une duché qui est procheinne 
Des montaingnes de Lombardie. (G. de Machaut, La Prise d'Alexandrie, 1396, v.1504-1514)

[Après tout cela, il prit congé du duc et de la compagnie et il partit tout content et jamais il ne s'arrêtera jusqu'au moment où il sera arrivé à Venise. Je vais maintenant mettre par écrit, sur ce parchemin, le chemin qu'il a parcouru. Il s'en alla en Carinthie, un duché à proximité des montagnes du nord de l'Italie.]

(8) Et avoit pour lors li fils dou roi Edouwart d'Engleterre, Edouwars, li ainnés de tous ses enfans, trese ans d'eage ou environ et fu la a celle feste creés et nonmés prinches de Galles; et l'en fu baillié la signourie et aministration, et en tint et entra lors en l'estat, et sera en avant nonmés prinches de Galles. Li contes de Hainnau et son oncle, li sires de Biaumont, furent mandé et priiet pour estre a celle feste et de l'ordenance dou Bleu Gertier, mais point n' i furent, car il estoient ensonniiet aillours; [...]. (Jean Froissart, Chroniques, ch. 173, 1400 ca.) personnages (DR), qui se trouvent enchâssés dans la narration. Dans ce cas, l'énonciateur $\mathrm{e}_{1}$ par rapport auquel doit être calculée l'ultériorité qu'implique l'emploi du futur n'est plus le narrateur (projeté dans le passé) mais un personnage. Nous distinguons deux cas de figure, selon la nature marquée ou non du DR concerné : les futurs employés en discours indirect (DI) et ceux figurant en discours indirect libre (DIL).

\subsubsection{Un décrochage énonciatif à l'intérieur du DI}

La présence du futur à l'intérieur d'un discours indirect peut être conforme à nos attentes, lorsque celui-ci est introduit par un verbe de parole employé au présent historique :

(9) Et l'andemain, quant il ert jorz,

Dient qu'il s'an voldront torner. (Lancelot, v. 5274-75)

[Le lendemain, lorsqu'il faisait jour, ils disent qu'ils voudront repartir.]

Même si les paroles rapportées font partie d'événements passés par rapport au moment d'énonciation du narrateur, le futur voldront ne surprend pas. Le décrochage énonciatif a bien eu lieu, mais il se situe dans le contexte gauche du futur, lors du passage d'une narration au passé (avec l'imparfait ert) au présent historique dient ; à l'intérieur du DI, la concordance des temps (dïent / voldront) est en revanche respectée.

La situation est toutefois différente lorsque le discours citant comporte un verbe conjugué au passé :

(10) demanda lor qu'il porra faire

des Troïens,... (Eneas, v. 3884-85)

[Il leur demanda ce qu'il pourra faire des Troyens.] 
(11) D’une chose se purpensa,

que l'aüstic engignera. (Aüstic, v. 93-94)

[Elle prit la décision qu'elle prendra le rossignol au piège.]

Dans le DI, qui est caractérisé par une homogénéité syntaxique et énonciative, tous les déictiques - dont les temps verbaux - sont normalement calculés par rapport à la situation d'énonciation du locuteur-énonciateur principal, donc par rapport au narrateur 10. Par conséquent, l'apparition des futurs porra (10) et engignera (11) à la suite des passés simples demanda (10) et se purpensa (11) marque un décrochage énonciatif qui se situe cette fois à l'intérieur même du discours rapporté. En effet, la valeur d'ultérieur qu'ils traduisent ne peut être comprise par rapport au présent d'énonciation $t_{0} d u$ narrateur mais doit être analysée en relation avec le moment d'énonciation $t_{1}$ du personnage (que nous pouvons alors assimiler à $\mathrm{e}_{1}$ ) auquel les paroles - ou les pensées - rapportées sont attribuées.

Un tel décrochage énonciatif entre discours citant et discours cité est normalement la règle uniquement dans le discours direct (DD), ce que soulignent d'ailleurs S. Azzopardi et J. Bres : «malgré la subordination syntaxique [...], l'ultériorité se calcule, comme dans le discours direct, à partir de l'acte énonciatif de $\mathrm{e}_{1}$, dont le discours est rapporté » (2011 : 59-60).

Cet emploi du futur dans des DI régis par un discours citant au passé n'est pas propre à l'AF. Il est également présent dans nos textes en MF, notamment dans des textes narratifs comme les Cent nouvelles nouvelles:

(12) Il fut beaucoup mercyé, ne doubtez, et des lors conclurent qu'il appellera sa niepce Conrard. (CNN, 172)

(13) Si fut ordonné qu'il demourra prestre. (CNN, 288)

Notons toutefois que le français médiéval connaît aussi, dans le même type de construction syntaxique, l'emploi d'un verbe au conditionnel, qui correspond alors à ce que l'on appelle en FM le futur dans le passé :

(14a) Al tierz jur dist qu'il baignereit. (Equitan, v. 273)

[Le troisième jour, il dit qu'il prendrait un bain.]

(14b) Il me dit que je l'avoye rompu et je luy respondy que non avoie et qu'il ne saroit monstrer. $(\mathrm{CNN}, 56)$

4 Nous pouvons enfin rappeler que l'emploi dialogique du futur dans un DI au passé s'est maintenu jusqu'en français contemporain, où on le rencontre en particulier dans le style journalistique. Le discours cité au futur peut alors même figurer entre guillemets ${ }^{11}$, et être marqué, dans ce cas, comme un véritable îlot textuel ${ }^{12}$, qui conserve les mots du locuteur-énonciateur $\mathrm{e}_{1}$.

\subsubsection{Un indice du DIL}

Alors que les textes narratifs en prose sont, pendant la période médiévale, caractérisés par un marquage très strict des frontières énonciatives (entre discours des personnages et discours du narrateur) et privilégient de ce fait les formes explicites du discours rapporté (DD et $\mathrm{DI}$ ), les textes versifiés du $12^{\mathrm{e}}$ siècle témoignent d'une certaine porosité des frontières énonciatives et accordent donc une place plus importante aux formes non marquées du DR. Les emplois dialogiques du futur - nécessitant un calcul par rapport au moment d'énonciation $t_{1}$ du personnage $e_{1}$ - peuvent alors aussi se rencontrer en DIL :

(15) $\mathrm{El}$ [Yseut] plore, il [le roi Marc] dit qu'ele se tese :

Ja nes mescrerra mais nul jor 
Por dit de nul losengeor ;

Allent et viengent a lor buens.

Li avoirs Tristran ert mes suens

Et li suens avoirs ert Tristrans.

N'en crerra mais Corneualans. (Tristan, v. 462-68)

[Elle pleure, il lui dit de se taire : plus aucun jour il ne les soupçonnera à cause des paroles de quelque menteur; qu'ils aillent et viennent à leur gré. Les biens de Tristan seront désormais les siens, et les siens seront à Tristan. Il ne se fiera plus aux Cornouaillais.]

(16) Et lors fu il asseürez

Qu'il ne pooit mes esgarer.

Qui que le doie conparer,

Ne finera tant que il voie

Le pin qui la fontainne onbroie,

Et le perron et la tormante

Qui grausle et pluet et tone et vante. (Yvain, v. 768-74)

[Alors il eut la certitude qu'il ne pouvait plus s'égarer. Quoi que cela doive lui coûter, il poursuivra sa route jusqu'à ce qu'il voie le pin ombrageant la fontaine, et le perron et la tourmente, qui déchaîne la grêle, la pluie, le tonnerre et le vent.]

Les futurs de ces deux occurrences ont des propriétés communes avec ceux de (9) à (11) : dans (15), le futur se situe, comme dans (9), à la suite d'au moins un verbe correspondant à un présent historique et témoignant donc déjà d'un décrochage énonciatif par rapport au moment d'énonciation $t_{0}$ du narrateur; dans (16), il est précédé, comme dans (10) et (11), d'au moins un verbe au passé: c'est alors ce futur, employé «à la place » du conditionnel, qui marque lui-même le décrochage énonciatif en question, le passage du calcul temporel par rapport à $t_{o}$ à celui par rapport au moment d'énonciation $t_{1} d u$ personnage $e_{1}$.

Mais, contrairement aux exemples de 2.2.1., les futurs de (15) et (16) ne sont pas subordonnés à un discours citant, et figurent donc bien à l'intérieur d'une forme interprétative du discours rapporté, le DIL. Cette absence de marquage explicite ne pose pas de problème d'interprétation ici, étant donné que nos deux DIL suivent directement un DI, donc une forme marquée du DR, correspondant à des paroles (15) ou à des pensées (16) rapportées.

Si les exemples de ce genre sont très rares dans la prose médiévale - vu son utilisation très limitée des formes non marquées du DR -, nous en trouvons toutefois une attestation dans les CNN. Reprenons (12) avec son contexte gauche :

(12a) [il] fut content de la servir et de l'accompaigner en tout ce qu'elle vouldra. Il fut beaucoup mercyé, ne doubtez, et des lors conclurent qu'il appellera sa niepce Conrard (CNN, 172)

L'interprétation dans le cadre du DIL est confirmée ici par le contexte droit de vouldra, qui marque la réponse (sous forme de remerciement) à cet énoncé. futur, l'analyse de ce dernier comme relevant du DIL (ou des pensées rapportées) est également envisageable:

(17) Dunc s'esteit la vieille levee,

triers une cortine est alee ;

bien purra oïr e veeir

ceo qu'ele cuveite a saveir. (Yonec, v. 267-70)

[Alors la vieille, qui s'était levée, s'est cachée derrière une tenture ; elle pourra bien

entendre et voir ce qu'elle a envie de savoir.] 
(18) En une chanbre les [les armes noires] anserre,

Que nus nes truisse ne ne voie,

Et fet a l'uis devers la voie

Les armes verz metre an presant,

Si les verront li trespassant.

Et se nus le demande et quiert,

Ne savront ou ses ostex iert. (Cligès, v. 4666-72)

[Il <Cligès> les enferme dans une chambre, afin que personne ne puisse les trouver ni les voir, et il fait mettre les armes vertes en évidence à la porte, du côté de la rue. Ainsi, les passants les verront, et si quelqu'un le demande ou le cherche, il ne saura pas où se trouve son logis.]

Dans (17) et (18), l'emploi du futur nous invite, une fois de plus, à modifier le calcul des déictiques de temps, étant donné que les procès évoqués renvoient à des événements passés par rapport au moment d'énonciation du narrateur $E_{1}$. Nous pouvons alors comprendre que ces futurs sont calculés par rapport au présent d'énonciation du personnage (qui devient dans ce cas $e_{1}$ ) et correspondent à des pensées rapportées, explicitant les intentions de la vieille (17) et de Cligès (18) : la vieille se cache derrière la tenture dans le but d'épier sa jeune maîtresse; Cligès met en évidence l'armure verte pour que l'on ne puisse pas trouver son logis.

Néanmoins, ce n'est pas là l'unique interprétation possible pour ces futurs. Il serait en effet tout aussi cohérent de les traiter comme des futurs historiques, permettant au narrateur de mettre en perspective les conséquences des actes de ses personnages : moi, narrateur, je sais que la vieille pourra ainsi épier sa jeune maîtresse (17), que, grâce à cette précaution, les passants ne trouveront pas le logis de Cligès (18)... Dans ce cas, les deux exemples se rapprochent de (5), où le narrateur commente également le comportement de son personnage :

(5) Erec fu foibles et quassez,

et cil fu auques respassez

de ses plaies et de ses cos.

Or fera Erec trop que fos

se tost conuistre ne se fet. (Erec, v. 4965-69)

Pour la période du moyen français, ces exemples peuvent être rapprochés de (19):

(19) Madame se alla mettre dedans le lict ou monseigneur devoit trouver sa

chambriere et droit là attendoit ce que Dieu luy vouldra envoyer $(C N N, 75)$

où le futur vouldra peut être l'indice d'un DIL assumé par le personnage $\left(e_{1}\right)$ mis en scène ou correspondre à un clin d'œil du narrateur (projeté au temps des événements racontés et endossant alors le rôle de $e_{1}$ ) à l'adresse de ses lecteurs.

Nous ne pensons pas qu'il faille à tout prix choisir, dans (17) à (19), entre ces deux interprétations: lorsqu'un énoncé susceptible de comporter un futur dialogique ne s'inscrit pas dans un dialogue comportant, par ailleurs, des formes marquées du DR, mais apparait, comme ici, directement dans la narration, son analyse comme DIL est souvent seulement envisageable mais nullement nécessaire pour préserver la cohérence du texte.

\section{Les valeurs modales du futur}

Issu d'une périphrase verbale à laquelle les linguistes ont attribué de nombreuses valeurs modales, le futur en $\mathrm{AF}$ et en MF a également conservé certaines de ces valeurs, même si l'AF et le MF font en même temps un usage assez intensif des verbes modaux comme 
vouloir, devoir, pouvoir. Les périphrases avec vouloir et devoir servaient d'ailleurs aussi à marquer la futurité, comme l'ont fait remarquer Buridant (2000: 363-64) et Martin \& Wilmet (1980 : 65-66). Mais quelles sont exactement les valeurs modales que ces formes peuvent exprimer? Est-ce que les grammaires et les études publiées jusqu'ici ont réussi à les identifier toutes précisément?

Notre corpus nous a offert un éventail assez large d'occurrences pour lesquelles on ne peut pas attribuer au futur une valeur temporelle ou exclusivement temporelle. Pour cette contribution, nous avons retenu deux emplois particuliers, dont l'un n'a été que partiellement évoqué par les grammaires et l'autre n'a pas encore fait l'objet d'une explication convaincante : le futur conjectural et le futur en énoncé interrogatif.

\subsection{Le futur épistémique, dit 'de conjecture'}

Ce type d'emploi, dit conjectural ou d'hypothèse probable, est attesté dans plusieurs langues romanes comme l'italien, l'espagnol et le français. Même si les grammaires de la langue ancienne mentionnent rarement cet emploi - l'étude de Wilmet sur l'indicatif (1970) n'en retient en effet que deux exemples ${ }^{13}$-, les occurrences sont nombreuses dans notre corpus à partir du $15^{\mathrm{e}}$ siècle. Le nombre de cas relevés dans nos textes en MF montre la productivité de cette construction à valeur conjecturale, en particulier avec le verbe estre à la $3^{\mathrm{e}}$ personne, suivi d'un adjectif comme bon ou d'un substantif comme heure ou temps.

(20) Nous avons esté a repoz

long temps et fait grant residence,

si sera temps, comme je pence,

que vers le Temple nous tirons ( $A A 1,86 \mathrm{v})$

(21) Aprés ceste ordonnance doncques

des articles de nostre foy,

il sera bon, comme je croy,

que nous les aillons publïer. (AA 1,16r)

(22) ELICE : Mere, à la porte, on appelle, nostre passe-temps est gasté.

CELESTINE: Regarde ma fille, possible sera-ce tel, qui le rabillera. (Céléstine, 149)

(23) MELıBÉE : Doux Amy Caliste, quel bruit est-ce que j'entens là dehors ? Je pense

ouyr le bruit de plusieurs mis en fuitte. Pour Dieu ayez soin de ta personne, car tu n'és pas seurement.

CALISTE : Ne te soucie, ma Deesse, je suis venu bien accompagné. Ce doivent estre les miens, qui sont Diables deschainez. Ils arrestent, et desarment autant qu'il en passe: quelqu'un leur sera eschappé, et seront allez apres pour le desarmer. ( Céléstine, 189-190)

(24) CALISTE : Malican? Malican? Malican ? Où sera ce malheureux ?

MALICAN : Me voicy Monsieur, qui garde ces chevaux. (Céléstine, 2)

Les occurrences relevées dans notre corpus montrent qu'effectivement cet usage de type épistémique n'est attesté en français qu'à partir du $15^{\mathrm{e}}$ siècle et qu'il concerne majoritairement le verbe être. En effet, comme le fait remarquer à juste titre Rocci, pour l'italien et le français, la lecture épistémique est favorisée par la classe aspectuelle du verbe (2000: 253) et, dans nos exemples, on relève majoritairement estre, un verbe de la catégorie stative. Par contre, quand le verbe appartient à la catégorie dynamique, d'autres éléments contextuels sont nécessaires afin de favoriser l'interprétation épistémique. 
50 La présence, dans nos extraits, d'énoncés du type «comme je pence » (20) et « comme je croy » (21), d'autres termes à valeur épistémique comme l'adverbe possible (au sens de peut-être) (22) et l'auxiliaire modal devoir (23), ou de la modalité interrogative (24) soulignent la nature évaluative ou interprétative (pour utiliser la terminologie de Rocci 2000) de ces futurs : le locuteur ne peut pas asserter avec certitude, ce qu'il affirme est le fruit d'une évaluation personnelle sur la base d'éléments cotextuels ou contextuels. Par ailleurs, le verbe à l'indicatif de ces incises indique bien que le procès est contemporain au moment de l'énonciation.

51 Cet emploi, encore vivant et bien attesté en italien et en espagnol modernes, se fait progressivement rare en français où il est réservé aujourd'hui à l'expression "ce sera " dont les études relèvent quelques cas, en général en discours direct et dans le langage courant, (l'exemple le plus cité étant celui de la Recherche de Proust, où la tante Léonie s'adressant à la servante, Françoise, dit "Les cloches sonnent. Ce sera pour Mme Rousseau »). Les linguistes ont déjà fait remarquer que dans tous ces emplois le futur peut être remplacé par le verbe devoir et c'est ce qui s'est passé dans l'histoire du français, comme le signale Rocci $(2000: 247)$, qui, comparant un roman italien du $20^{\mathrm{e}}$ siècle et sa traduction en FM, montre que tous les futurs épistémiques de l'italien ont été rendus par le traducteur par une périphrase avec le verbe devoir (comme dans 23).

52 D’après une étude de Bertinetto (1986 : 495-498) sur l'italien, la précocité des emplois du futur conjectural, ainsi que leur fréquence dans la langue parlée (ou plus précisément dans l'oral représenté pour les époques anciennes), témoignerait de la priorité des valeurs modales du futur sur les valeurs temporelles. Même s'il est difficile d'appliquer la même analyse au français ancien, étant donné qu'en français le futur épistémique a connu une évolution très différente, on retiendra ici que ces emplois sont bien plus fréquents en MF et en français pré-classique et qu'ils présentent une palette plus large du point de vue des formes (en FM, cela ne concerne que les verbes être et avoir et majoritairement la $3^{\mathrm{e}}$ personne). Nous soulignons aussi que tous ces exemples ont été relevés dans le dialogue, dans un registre qui, s'il n'est pas populaire, relève de la conversation quotidienne et de l'oral. La haute fréquence dépend probablement aussi du type de texte ; il n'est pas étonnant en effet que le seul à en avoir relevé des exemples soit Wilmet qui a travaillé, comme nous, sur un corpus de textes dramatiques.

53 Les valeurs modales précèdent-elles les valeurs temporelles? Les résultats de notre enquête partielle nous incitent à la prudence, les premières apparitions de formes de futur en français, (comme le célèbre «si salvarai eo cist meon fradre» des Serments de Strasbourg, $9^{\mathrm{e}} \mathrm{s}$.) montrent qu'il est difficile, pour ces occurrences, de distinguer entre une valeur purement temporelle et une valeur purement modale. Nous y reviendrons à la fin de notre étude.

\subsection{Le futur en proposition interrogative}

54 Notre recherche dans le corpus en $\mathrm{AF}$ et en MF a fait ressortir un autre emploi très fréquent dans les dialogues et monologues dramatiques qui apparaît en énoncé interrogatif et qui ne peut pas toujours se comprendre en termes d'ultériorité par rapport au moment de l'énonciation, même si, comme l'a bien montré Fleischman (1982), depuis le latin la catégorie de la futurité est étroitement liée avec diverses modalités (obligation, volition, croyance) et qu'il peut donc être difficile de déterminer, pour certaines 
occurrences, si c'est la valeur modale qui prime ou la valeur temporelle. En voici quelques occurrences :

(25) Quant Enyde les ot veüz,

toz li sans li est esmeüz,

grant peor ot et grant esmai.

«Lasse, fet ele, que ferai?

Ne sai que die ne que face,

que mes sires mout me menace

et dit qu'il me fera enui

se je de rien paroil a lui. » (Erec, v. 2959-66)

[Quand Enide les aperçut, elle devint blême ; elle ressentit une grande frayeur et un grand trouble. «Malheureuse, dit-elle, que dois-je faire? Je ne sais ni quoi dire ni quoi faire, car mon époux me menace fort et me dit qu'il me le fera payer si je lui dis quoi que ce soit. »]

Le vers 2963 montre bien que l'hésitation de la locutrice est située au moment présent $t_{\text {o }}$.

Nous avons observé ce même phénomène dans les dialogues en MF. Dans les exemples suivants, extraits d'un texte dramatique de la fin du $15^{\mathrm{e}}$ siècle, le Mystère des Actes des Apôtres, la présence de didascalies et/ou la situation scénique prouvent que les questions que se posent les locuteurs portent bien sur la situation d'énonciation présente au moment $t_{0}$; le futur concerne alors plutôt la modalité de l'action. Si le futur dit «immédiat » est fréquent en MF, de nombreux emplois de futur en interrogative ne semblent pas traduire l'ultériorité par rapport à l'énonciation, mais exprimer plutôt une modalité particulière (pouvoir, vouloir, devoir) :

(26) Serpent mauldict, te tairas tu, faulx diable ? $(A A, 1)$ [Serpent maudit, est-ce que tu veux bien te taire, faux diable !]

(27) Sa! Entrerons nous? (AA 1, 43r) (ils sont devant la porte) [Est-ce que nous pouvons entrer?]

7 Ces emplois sont également attestés dans les dialogues à l'intérieur d'un récit :

(28) Pendant ce temps, les gcntilz hommes de l'ostel ou ce nouvel marié demouroit vindrent hurter a l'huys de ceste chambre et apporterent le chaudeau. Ilz hurterent beaucop sans ce que ame respondist. L'espousée en estoit bien excusée, et l'espousé n'avoit pas cause de trop hault caqueter : «Et qu'est ce cy ? dirent ilz, et n'ouvrirez vous pas l'huys? (CNN, 198) [n'avez-vous pas l'intention d'ouvrir la porte ?]

(29) celle qui avoit sa pitance dist : «ça, voisine, en yrons nous? - Voire, dit l'autre, s'en va l'on ainsi? » $(C N N, 522)$ [allez , ma chère voisine, pouvons nous partir ?]

Dans certains cas, si la valeur temporelle est faible ou nulle, il apparait clairement que le verbe au futur traduit soit un acte illocutoire comme la demande d'autorisation (30), soit une modalité particulière (déontique, aléthique ou épistémique), qui le rapproche de constructions à verbe modal, comme, par exemple, pouvoir (31), devoir (32) ou falloir (33) :

(30) AMON : Prevost, le ferons nous mourir

et finer douloureusement?

Baillez vostre consentement

sans plus, il sera depesché ! (AA 3, 90v) [pouvons-nous le tuer pour qu'il finisse ses jours dans la souffrance? Donnez-nous votre accord et il en sera fait de lui]

(31) Comme lairra Dieu mourir ces bonnes gens de Compieigne, qui ont esté si loyaulx a leur seigneur? (PJA, 14 mars) [Comment Dieu peut-il laisser mourir ces bonnes gens de Compiègne?]

(32) Plebere: Ma fille Melibee, que me veux tu ma mie ? que fais tu là seulette ? qu'as tu à me dire ? iray-je là hault à toy ? [est-ce que je dois monter là-haut ?] MELIBEE : Non, mon pere, n'en pren ja la peine (Célestine, 259v) 
(33) et son tresennuyé pere destort ses mains, ses cheveulx detire par la grand rage de ce nouvel courroux. Que vous diray je? toute la grand triumphe qui en cest hostel souloit comblement abunder est par ce cas abatue et ternye, et en amere et subite tristece a la male heure [convertye] $(C N N, 32)$ [Que puis-je vous dire ? Que faut-il que je vous dise ?] une valeur modale et faire intervenir un jugement du locuteur par rapport au contenu de l'énoncé. Il est cependant difficile de définir cette valeur modale, en absence de verbe modal ou d'un autre élément modalisateur. Si on peut admettre que dans les exemples (25), (32) la valeur exprimée est l'obligation (« que dois-je faire?»), ailleurs, on dirait que le locuteur demande la permission de faire quelque chose (27), ou qu'il s'agit plutôt d'une possibilité que le locuteur envisage sans trop y croire (26), (28), (29). formes du futur en MF, nous citons encore deux occurrences extraites de notre corpus, l'une en $\mathrm{AF}$ :

(34) « Que feras tu ? Conselle toi.

- Sire, j'am Yseut a mervelle,

[...]

De lié laisier parler ne ruis,

Certes, quar faire ne le puis. » (Tristan, v. 1400-08)

[Que vas-tu/peux-tu faire ? Réfléchis. - Seigneur, j’aime éperdument Yseut... Je ne veux pas qu'on me dise de l'abandonner, car, assurément, je ne suis pas en mesure de le faire.]

l'autre en MF :

(35) Que feray je triste, et chetive? De retourner arriere, il n'y a nul acquest: et si n'est la perseverance sans inconvenient. Quoy donc marcheray je, ou si je doy retourner (La Célestine) 
car, dans les deux, la forme au futur est soit coordonnée (35) à une périphrase modale, soit reprise par une périphrase modale (34).

Il est aussi intéressant de remarquer que l'un des rares grammairiens du $16^{\mathrm{e}}$ siècle à mentionner brièvement le sémantisme de ce paradigme, Jean Bosquet, associe aussi les formes de futur à l'idée d'obligation / nécessité : «Que signifie aussy le futur ? La chose que l'on doit faire au temps advenir, comme je parleray, je chanteray» (1586:76). Ce qui prouve que la valeur temporelle (au temps advenir) est étroitement liée à la modalité (doit faire), comme le fera remarquer aussi Nicolas Beauzée au $18^{\mathrm{e}}$ siècle, en comparant le latin laudaturus sum et la périphrase française « je dois louer » tout en insistant sur le fait qu'ils marquent la postérité de l'action par rapport moment de l'énonciation. (1767, I, 457-458).

\section{Conclusions}

Dans le cadre de cette étude, nous avons essayé de rendre compte de quelques emplois spécifiques - dialogiques et modaux - du futur dans la langue médiévale. Il s'agit de futurs dont la présence est liée à des contextes d'emplois et/ou à des contraintes (sémantiques ou syntaxiques) particuliers.

68 Si nous avons ainsi pu constater la précocité des emplois dialogiques du futur en français - ils sont attestés dès l'AF -, nous avons aussi vu que ces emplois apparaissent en premier lieu dans des textes narratifs dans lesquels le narrateur manifeste clairement sa présence et sa subjectivité et fait preuve d'une grande souplesse (voire d'une grande modernité) dans le traitement des paroles de ses personnages, en accordant une place non négligeable aux formes interprétatives du DR.

Grâce à la rupture énonciative dont il témoigne par rapport au moment d'énonciation $t_{0}$ du narrateur, le futur dialogique permet ainsi au narrateur :

- soit de se projeter, à l'intérieur même de la narration, au temps passé $t_{1}$ des évènements racontés, en entraînant son récepteur (qu'il soit auditeur ou lecteur) avec lui dans cette même période (futur historique);

- soit de marquer, dans un DR au style indirect (DI ou DIL), le passage de $t_{o}$ au moment d'énonciation $t_{1}$ de son personnage et de contribuer, par ce moyen, à la mise en scène des paroles ou des pensées de celui-ci.

Dans les deux cas, le futur dialogique tend à effacer la distance temporelle séparant $t_{1} d u$ moment d'énonciation $t_{0} d u$ narrateur; il «rapproche» donc l'auditeur-lecteur des actions et des paroles (ou pensées) narrées et fait de lui un témoin fictif privilégié de ces événements passés.

71 Les emplois du futur évoqués dans la deuxième partie de cette étude nous ont permis de montrer que la présence de valeurs modales est encore très forte à l'époque tardomédiévale et pré-moderne ( $15^{\mathrm{e}}$ et $16^{\mathrm{e}}$ siècles). Si le futur de conjecture n'est pas très fréquent dans notre corpus, le futur modal en interrogative est par contre bien représenté. Comme le futur dialogique, ces emplois modaux sont tous les deux contraints : s'ils apparaissent uniquement en dialogue, le premier privilégie les verbes statifs à la P3, l'autre se trouve toujours dans des énoncés à modalité interrogative.

Quand ces conditions sont vérifiées, la forme de futur peut être interprétée par le locuteur autrement que comme un indice temporel. 
été remplacé par une périphrase avec devoir; alors qu'en interrogative, le futur a été complètement remplacé soit par la futur périphrastique, soit par des périphrases avec les verbes pouvoir et devoir.

Il nous semble qu'on pourrait postuler, en suivant aussi ce que suggèrent Patard, De Mulder et Grabar dans une étude récente (sous presse), la permanence de valeurs modales encore très forte en français médiéval, (sans oublier le caractère prioritairement modal du futur, affirmé pour l'italien, par Parisi, Antinucci \& Crisari 1975 et Bertinetto 1986). Pendant une longue période en français, les formes de futur synthétique signaleraient simplement une inférence et le contexte et/ou les adverbes établiraient la référence temporelle (future ou autre).

D'un point de vue diachronique, on pourrait expliquer ce sémantisme essentiellement modal par la dérivation de la forme périphrastique latine qui véhiculait, dès le début, des valeurs modales: obligation (Thielman 1885), obligation / nécessité (Fleischman 1982), possibilité (Pinkster 2013), etc. A partir de ces valeurs modales se serait développée la capacité à exprimer la futurité d'un événement, présente dès les premières occurrences en français. Les exemples relevés dans notre corpus semblent montrer que le sémantisme ancien et originaire de la périphrase latine demeure dans les emplois de futur et notamment le fait d'exprimer la possibilité ou l'obligation de réaliser une action (puis-je, dois-je, etc.). La modalité et la temporalité sont étroitement liées jusqu'à l'apparition du futur périphrastique ( $15^{\mathrm{e}}$ siècle) qui assume progressivement quelques-unes des valeurs qui caractérisaient jusque-là le futur synthétique. Ce dernier se trouve ainsi dépouillé de certaines fonctions et voit son usage restreint à des contextes particuliers.

\section{BIBLIOGRAPHIE}

AUTHIER-REVUZ, J., 1992, 1993, « Repères dans le champ du discours rapporté », L'Information grammaticale 55 et 56, p. 38-42 et p. 10-15.

AZZOPARDI, S., 2011, Le futur et le conditionnel : valeur en langue et effets de sens en discours. Analyse contrastive espagnol / français, Thèse de doctorat, Université de Montpellier 3.

AZZOPARDI, S., BRES, J., 2011, « Temps verbal et énonciation. Le conditionnel et le futur en français : l'un est dialogique, l'autre pas (souvent) », Cahiers de Praxématique 56, p. 53-76.

BEAUZEE, N., 1767, Grammaire générale, ou Exposition raisonnée des éléments nécessaires du langage pour servir de fondement à l'étude de toutes les langues, Paris, Barbou, 2 vol.

BENVENISTE, E., 1974, Problèmes de linguistique générale, Paris, Gallimard.

BERTINETTO, P.-M., 1986, Tempo, aspetto e azione nel verbo italiano. Il sistema dell'indicativo, Firenze, Accademia della Crusca.

BOSQUET, J., 1586, Elemens ou institutions de la langue françoise, Mons.

BRES, J., 2009, « Dialogisme et temps verbaux de l'indicatif », Langue française 163, p. 21-39.

BURIDANT, C., 2000, Grammaire nouvelle de l'ancien français, Paris, Sedes. 
BYBEE, J., PERKINS, R., PAGLIUCA, W., 1994, The Evolution of Grammar : Tense, Aspect and Mood in the Languages of the World, Chicago, University of Chicago Press.

FLEISCHMAN, S., 1982, The Future in Thought and Language. Diachronic Evidence from Romance, Cambridge, Cambridge University Press.

LANLY, A., 1993, « Retour au conditionnel » dans R. Marchal et B. Guidot (éds), Lorraine Vivante, Mélanges Jean Lanher, Presses Universitaires de Nancy, p. 371-376.

LANLY, A., 1996, Deux problèmes de linguistique française et romane. I. Le conditionnel en -rais (et le futur en -rai). II. Le verbe aller et ses frères romans, Paris, Champion.

ARNAVIELLE, 1995, Compte-rendu de Lanly, (1993), dans Revue des Langues Romanes, 99, 1, p. 186-188.

MARNETTE, S., 1998, Narrateur et points de vue dans la littérature française médiévale : Une approche linguistique, Berne, Peter Lang.

MARTIN, R., WILMET, M., 1980, Syntaxe du moyen français, Bordeaux, Sobodi.

MÉNARD, Ph., 1994, Syntaxe de l'ancien français, Bordeaux, éd. Bière.

MOIGNET, G., 1984, Grammaire de l'ancien français, Paris, Klincksieck.

PARISI, D., ANTINUCCI, F., CRISARI, M., 1975, « Dovere, potere e volere e il futuro dei verbi », Studi per un modello del linguaggio, Roma, CNR, p. 238-270.

PATARD, A., DE MULDER, W., GRABAR, N. (sous presse), « Le futur synthétique a-t-il eu un sens intentionnel?».

PINKSTER, H., 2013, « The periphrastic future and conditional; present for future », dans J. N. Adams (éd.), Social variation and the Latin language, Cambridge, Cambridge university press, p. 652-673.

RIEGEL, M., PELLAT, J. C., RIOUL, R., 1996, Grammaire méthodique du français, Paris, PUF.

ROCCI, A., 2000, « L'interprétation épistémique du futur en italien et en français : une analyse procédurale », Cahiers de Linguistique française 22, p. 241-274.

ROSIER, L., WILMET, M., 2003, « La 'concordance des temps' revisitée ou de la 'concordance' à la 'convergence' ", Langue française 138, p. 97-110.

THIELMAN, Ph., 1885, « Habere mit dem Infinitiv und die Entstehung des romanischen Futurums ", Archiv für Lateinische Lexicographie und Grammatik mit Einschluss des älteren Mittellateins 2, 48-89, 157-202.

WILMET, M., 1970, Le système de l'indicatif en moyen français, Genève, Droz.

\section{NOTES}

1. Pour connaître une position discordante sur la formation du Futur II, voir A. Lanly (1993 et 1996), et les réserves d'Arnavielle (1995: 186-188) et Buridant (2000: 264)

2. Benveniste (1974), Fleischman (1982), Bybee et al. (1994).

3. Parmi les nombreuses études sur ce tiroir verbal citées en bibliographie, nous retiendrons ici : Bertinetto (1986), Vet (1994), Rocci (2000), Bres (2009) Azzopardi, Bres (2011), Patard, De Mulder, Grabar (sous presse).

4. A l'exception des Lais de Marie de France, qui ont été dépouillés à la main (éd. $\mathrm{K}$. Warnke, trad.

L. Harf-Lancner, Paris, Librairie générale française, 1990). 
5. Les manuels de l'ancien français, qui accordent peu de place aux différentes valeurs du futur, ne mentionnent pas le futur historique. Martin \& Wilmet (1980) en parlent toutefois pour la période du moyen français : «Le MF connaît déjà le futur « historique », fondé sur un décalage de l'actualité dans le passé (comparer au présent historique [...])» (1980:85).

6. Selon son contexte d'emploi, ert peut correspondre à la troisième personne du futur du verbe estre (< latin erit) - c'est le cas dans (2) - ou la troisième personne de l'imparfait du verbe estre (< latin erat), comme dans (1).

7. «Le présent historique qui d'une manière expressive rend présent et met sous les yeux un événement passé est très répandu en $\mathrm{AF}$, notamment dans la littérature narrative. " (Ménard, 1994 : 138), cf. aussi Moignet (1984: 255).

8. «les lais et les romans en vers sont des textes du je» (Marnette, 1998: 32), ils «ont donc davantage tendance que les autres textes à mettre en scène un 'je narrateur' » (ibid. : 34).

9. M. Wilmet (1970: 178), qui a travaillé à partir d'un corpus dramatique en MF, en relève deux exemples qui n'emportent pas l'adhésion. Pour qu'il y ait un futur historique, il faut en effet un narrateur, et les personnages de théâtre font rarement de longs récits au passé.

10. Le DI correspond « à une phrase normale de la langue » (Authier-Revuz, 1993:11) et «n'a qu'un cadre de repérage pour les déictiques, celui de la situation effective d'énonciation $\mathrm{E}$ » (ibid. :12).

11. Si l'on en croit l'exemple cité par Rosier \& Wilmet $(2003: 108)$ : Il a réaffirmé que « toutes les statues seront détruites conformément au décret du mollah Omar " (La Dernière Heure 02/03/2001:92).

12. Ce terme est emprunté à Authier-Revuz (1992)

13. Ils sont extraits de textes dramatiques de la fin $\mathrm{du} 15^{\mathrm{e}}$ siècle : « Il y aura quelque nouvelle » ( Farces, éd. Cohen, LI, 258-62) et « Ce sera quelque dyablerie » (Sotties, éd. Picot, X, 237-41).

14. Au sujet de la périphrase avec le verbe aller, on verra: De Mulder W. et Vanderheyden A. (2008), Vetters C. et Lière A. (2009).

\section{RÉSUMÉS}

Cet article est consacré à deux types d'emplois du futur dans lesquels ce temps verbal ne peut s'interpréter en termes d'ultériorité par rapport au moment d'énonciation. Le premier est représenté par des futurs que nous pouvons appeler dialogiques, à l'intérieur desquels nous distinguons le futur historique et le futur employé en discours indirect et indirect libre. Se pose alors, entre autres, la question du moment d'énonciation par rapport auquel l'ultériorité exprimée par le futur doit être envisagée. Le deuxième type d'emplois concerne des futurs qui ont en premier lieu une valeur modale: le futur épistémique, dit 'de conjecture', et le futur employé dans l'interrogation, qui traduit une hésitation du locuteur, un doute sur la réalisation du procès au moment même de l'énonciation. L'étude s'appuie sur un corpus d'ancien et de moyen français, composé de textes narratifs en vers $\mathrm{du} 12^{\mathrm{e}}$ siècle et de textes narratifs (en prose) et dramatiques (en vers) du 15e siècle. Elle met en évidence la précocité des emplois dialogique et conjectural $\mathrm{du}$ futur en français ainsi que les contraintes d'emplois propres à ces futurs, et souligne par ailleurs l'importance du sémantisme modal du futur synthétique pendant l'ensemble de la période médiévale, ce qui peut s'expliquer par la valeur modale initiale de la périphrase latine dont il est issu. 
This paper analyses two different uses of the future tense for which it cannot be interpreted in terms of ulteriority in reference to speech time. The first use consists of future forms that could be called "dialogical", among which we differenciate the historical future and the future used in reported speech and free indirect speech. One of the questions which arises is how to identify the reference time in relation to which the ulteriority expressed by the future must be considered. The second type of use includes future forms that have a modal value: the epistemic futur called 'conjectural' and the future used in interrogative sentences, which reflects the speaker's hesitation or doubt about the unfolding of the process expressed by the verb. The present study is based on a corpus of old and Middle French, which comprises $12^{\text {th }}$-century narrative texts in verse and $15^{\text {th }}$-century narrative texts (in prose) and dramatic texts (in verse). It highlights how early the dialogical and conjectural uses of the future in French, as well as the specific constraints that characterise them. Moreover, this study emphasises the importance of the modal meaning of the synthetic future throughout the Middle Ages, which can be explained by the modal interpretation of the latin periphrasis from which it derives.

\section{INDEX}

Mots-clés : temps futur, ancien français, moyen français

Keywords : future tense, old French, Middle French

\section{AUTEURS}

\section{EVELYNE OPPERMANN-MARSAUX}

Université de la Sorbonne Nouvelle - Paris 3 / EA 7345 CLESTHIA

\section{GABRIELLA PARUSSA}

Université de la Sorbonne Nouvelle - Paris 3 / EA 7345 CLESTHIA 